

\section{NOTICE}

This report was prepared as an account of work snossored by an agency of the United States Government. Neither the United States nor any ajorcy thercof, nor any of their cinployees, makes any iarranty, expressed or inplied, or assumes any legal liability or responsibility for any $t:=d$ party's use or the results of such use of an $y$ information, apparatus, product or process disclosed in this report, or represents that its use by such third party would not infringe privately owned rights.

Printed in the United States of Arerica Avai"iable from

National Techn,cal Information Service U.S. Department of Comnerce 5285 Port Royal Road Springfield, VA 22161

N'IS Price codes Printed copy: $\quad \mathrm{A} 02$ Microfiche copy: A01 


\title{
Soft X-ray Measurement of Internal Tearing Mode Structure in a Reversed-Field Pinch
}

\author{
George Chartas $(a)$ \\ Samuel Hokin \\ Department of Physics, University of Wisconsin, Madison, WI 53706
}

September 16, 1991

\begin{abstract}
The structure of internally resonant teering modes has been studied in the Madison Symmetric Torus reversed-field pinch with a soft $x$-ray detector system consisting of an imaging array at one toroidal location and several detectors at different toroidal locations. The toroidal mode numbers of $m=1$ structures are in the range $n=-5,-6,-7$. The modes propagate with phase velocity $v=1-6 \times 10^{6} \mathrm{~cm} / \mathrm{s}$, larger than the diamagnetic drift velocity $v_{d} \sim 5 \times 10^{5} \mathrm{~cm} / \mathrm{s}$. Phase locking between modes with different $n$ is manifested as a beating of soft $\mathrm{x}$-ray signals which is found to be strongest near the resonant surfaces of the modes $(r / a=0.3-0.5)$.
\end{abstract}

(a)Present address: Harvard-Smithsonian Astrophysical Observatory, Cambridge, MA 02138 
The reversed-field pinch (RFP) differs from the tokamak primarily in the reduced toroidal magnetic field (for a given toroidal current) and the large resultant shear of magnetic field lines which provides stability against pressure-driven modes $[1,2]$. Due to the reduced toroidal field, the safety factor $q(r) \equiv$ $r B_{\phi} / R B_{\theta}$ differs greatly from the tokamak ( $r$ is the minor radial coordinate, $R$ is the major radius, $B_{\phi}$ and $B_{\theta}$ are the toroidal and poloidal fields, repectively). Fig. 1 displays a comparison of a tokamak $q$ profile [3] and an RFP $q$ profile calculated using the Bessel Function Model [4] and aspect ratio $R / a=1.5 / 0.52$ of the Madison Symmetric Torus (MST).

The radial locations $r_{s}$ of resonant surfaces, around which the radial displacement due to tearing mode instability is large, are given by the condition $q\left(r_{s}\right)=-m / n$, where $m$ and $n$ are integer poloidal and toroidal mode numbers respectively, and $B_{\phi}, B_{\theta}$ are defined to be positive in the plasma core. Unlike the tokamak, which tends to have a single $m=1, n=-1$ internal resonant surface, the RFP has several internally resonant surfaces with $n \sim-2 R / a$ (Fig. 1). The nonlinear interaction of neighboring modes in the RFP limits their growth [5] and is believed to be responsible for toroidal field generation, which often occurs in sudden 'discrete dynamo' events $[6,7]$.

This letter focuses on measurements of tearing mode oscillations in the MST RFP by means of a 32-chord soft $x$-ray (SXR) imaging array at one toroidal location and four centrally viewing SXR chords at other toroidal locations (Fig. 2). The study of internal modes using SXR diagnostics has been limited in previous work on RFP's due to the restricted access of poris. ZT-40M [8] and HBTX [9] were not equipped with enough SXR chords to implement tomography, while Repute-1 [10] had only two chord arrays with a total of 18 detectors. The MST setup, on the other hand, is well-suited for tomographic reconstruction of SXR emission at one toroidal location with simultaneous measurement of toroidal mode numbers.

The first observation of an $m=1$ oscillation in SXR signals in an RFP was made in ZT-40M [8]. The $m=1$ nature of the mode was inferred from a measurement of the relative phases of signals from an array of 8 surface barrier diode (SBD) detectors. An estimate of the toroidal mode number was obtained by measurement of the relative phase between two toroidally separated SBD signals. However, it could not be determined whether the mode was internally or externally resonant, and no inference of the radial structure of the mode was possible. The resonant surfaces in ZT-40M are closely spaced due to the large aspect ratio of the device $(R / a=5.7)$, which is expected to cause saturation of modes at 
lower relative amplitudes than in the case of MST, which has smaller aspect ratio and therefore larger separation between resonant surfaces. This is thought to account for the 'discrete dynamo' toroidal flux generation events on MST [7] which are much stronger than observed on other RFP experiments.

The SXR detectors on MST consist of SBD's filtered with 7.6 $\mu \mathrm{m}$ Be filters and collimated to optimize the spatial resolution of the imaging array. Measurements made with other filter materials as well as impurity simulations indicate that the filtered SXR spectrum is dominated by oxygen line radiation. The Maximum Entropy Method [11,12] (MEM) is used to reconstruct the emissivity profiles from the set of 32 SBD signals. Since the amplitude of the $m=1$ component of emissivity is much smaller than the background $(m=0)$ component, we performed a mode decomposition of the reconstructed profiles by using a Fourier-Bessel expansion. This was useful for determining the evolution of the $m=1$ amplitude at different radii.

The $m=1$ modes manifest themselves on SXR signals as oscillations with frequencies in the range $10-40 \mathrm{kHz}$. These are often termed 'precursors' since they precede a sawtooth crash. The rotation of the $m=1$ helix as it propagates past the imaging array is shown in Fig. 3 for a discharge with plasma current $I=350 \mathrm{kA}$ and $F \equiv B_{t}(a) /\left\langle B_{t}\right\rangle=-0.15$. In Fig. 4 we display signals, digitally high-pass filtered to emphasize the oscillations, measured from SBD's located at different toroidal angles (Fig. 2). Signals from detectors at $\phi=300^{\circ}$ and $\phi=330^{\circ}$ are shifted in phase by $360^{\circ}$, leading one, at first, to conclude that $|n|=360 / 30=12$. However, a 'double frequency effect' occurs on centrally viewing chords, which is displayed schematically in Fig. 4. Taking this into account, one finds $|n|=6$ in this case. Coherent $m=1$ modes are measured by this method as well as by edge magnetic coils to have $|n|$ in the range $5,6,7$.

The phase velocity of the mode is $v=\omega / k$ where $\mathbf{k}=\hat{\theta} \mathrm{m} / r+\hat{\phi} n / R$. For the observed range of frequencies and $n$ values, $v=1-6 \times 10^{6} \mathrm{~cm} / \mathrm{s}$, larger than the diamagnetic drift velocity $v_{d}=T / B a \sim$ $5 \times 10^{5} \mathrm{~cm} / \mathrm{s}$, where the plasma temperature $T \sim 250 \mathrm{eV}$. Simultaneous measurement of thr direction of poloidal and toroidal rotation indicates that the mode is internal, ie. $n=-6$ when $m$ is positive: the imaging array typically reveals rotation in the $+\theta$ direction when the toroidal array shows propagation in the $-\phi$ direction, corresponding to ion diarnagnetic drift. However, rotation in the $-\theta,+\phi$ direction has also been observed. 
A revealing phenomenon may be observed by viewing SXR chords of different impact parameter (Fig. 5). A beating of the signals is clearly evident and particularly strong on those labeled SXR-2 and SXR-3 which have impact parameters $r / a=0.48$ and $r / a=0.39$ respectively. A simple explanation of the origin of this beating follows. Consider two unstable modes with $n=-5$ and $n=-6$. Their phase velocity has been measured with edge magnetic coil arrays [13] to be equal ('phase locking'), ie. $v=\omega_{5} / k_{5}=\omega_{6} / k_{6}$ giving $\Delta \omega / \omega=\Delta n / n \sim 1 / 6$. The phase locking of two modes will therefore produce beats with the number of oscillations in one beat period approximately equal to the toroidal mode number $n$. This is observed on the signals labeled SXR-2 and SXR-3. A complete cancellation (beat node) occurs when the waves are $180^{\circ}$ out of phase and of equal amplitude. We expect this to occur at the location where the radial displacement functions of the $n=-5$ and $n=-6$ modes are of equal amplitude. Chords SXR-2 and SXR-3 are in the vicinity of the $q=1 / 6$ and $q=1 / 5$ resonant surfaces calculated from RFP equilibrium models [4,14] (Fig. 1).

The presence of the phase locking is not easily distinguished in reconstructed SXR emissivity profiles since it is a localized disturbance of small amplitude compared to the total emissivity. We have applied a Fourier-Bessel expansion of the reconstructed profiles to isolate individual $m$ components of emission. The beating of the $m=1$ component is strongest at $r / a \sim 0.5$, corresponding to the impact parameter of chord SXR-2.

The localized phase locking observed in MST differs from the localized 'slinky mode' observed in the OHTE device operated with a thin resistive shell [15]. The appearance of the slinky mode in OHTE coincided with the 'turning off' of internally resonant $m=1$ modes. This was thought to be due to the flattening of the $\mu=\mathbf{J} \cdot \mathbf{B} / B^{2}$ profile caused by the magnetic field line mixing introduced by the slinky. In MST, the internal modes do not turn off with the onset of phase locking; in fact, the modes often continue to rotate even after the discrete dynamo event which rearranges the magnetic field topology.

In summary, the dominant internal $m=1$ modes present in the MST RFP are measured with soft $\mathrm{x}$-ray diagnostics to have toroidal mode numbers in the range $-5,-6,-7$. These modes propagate with phase velocities in the range $1-6 \times 10^{6} \mathrm{~cm} / \mathrm{s}$, larger than the diamagnetic drift velocity, and phase lock together to produce beats on SXR precursor signals. The beat modulation of the precursor oscillations is found to be largest at $r / a=0.3-0.5$, which is the expected region of the $n=-5,-6,-7$ resonant surfaces. 
This work was supported by the US Department of Energy.

\section{References}

[1] C. Mercier, Nuclear Fusion 1, 47 (1960).

[2] H.A.B. Bodin and A.A. Newton, Nucl. Fusion 20, 1255 (1980).

[3] H. Soltwisch, W. Stodiek, J. Manickam, and J. Schluter, in Plasma Physics and Controlled Nuclear Fusion Research, 1986, (IAEA, Vienna, 1987), Vol. 1, p. 263.

[4] J.B. Taylor, Rev. Mod. Phys. 58, 741 (1986).

[5] J.A. Holmes, B.A. Carreras, T.C. Hender, H.R. Hicks, V.E. Lynch, Z.G. An, and P.H. Diamond, Phys. Fluids 28, 261 (1985).

[6] G. Chartas, Ph.D. thesis, University of Wisconsin (1991).

[7] S. Hokin, A. Almagri, J. Beckstead, G. Chartas, N. Crocker, M. Cudzinovic, D. Den Hartog, R. Dexter, D. Holly, S. Prager, T. Rempel, J. Sarff, E. Scime, W. Shen, C. Spragins, C. Sprott, G. Starr, M. Stoneking, C. Watts, and R. Nebel, Phys. Fluids B 3, 2241 (1991).

[8] G.A. Wurden, Phys. Fluids 27, 551 (1984).

[9] R.J. Hayden and B. Alper, Plasma Phys. Controlled Fusion 31, 193 (1989).

[10] N. Asakura, Y. Nacayama, S. Shinohara, H. Toyama, and K. Miyamoto, Nucl. Fusion 29, 893 (1989).

[11] J.E. Shore and R.W. Johnson, IEEE Trans. on Inf. Theory IT-26, 26 (1980).

[12] A. Holland and G.A. Navratil, Rev. Sci. Instrum. 57, 1557 (1986).

[13] A. Almagri, S. Assadi, J. Beckstead, G. Chartas, N. Crocker, D. Den Hartog, R. Dexter, S. Hokin, D. Holly, E. Nilles, S. Prager, T. Rempel, J. Sarf, E. Scime, W. Shen, C. Spragins, J. Sprott, G. Starr, M. Stoneking, and C. Watts, in Proceedings of the International School of Plasma Physics Workshop on Physics of Alternative Confinement Schemes, Varenna, Italy, 1990, edited by S. Ortolani and E. Sindoni (Società Italiana di Fisica, Bologna, 1991), p. 223. 
[14] W. Shen and J.C. Sprott, Phys. Fluids B 3, 1225 (1991).

[15] R.J. LaHaye, P.S. Lee, M.J. Schaffer, T. Tamano, and P.L. Taylor, Nucl. Fusion 28, 918 (1988). 


\section{Figure Captions}

Figure 1 Profiles of $q(r)=r B_{\phi} / R B_{\theta}$ for a tokamak and an RFP with aspect ratio $R / a=3$. The RFP case is calculated using the Bessel Function Model with $\Theta=B_{p}(a) /\left\langle B_{t}\right\rangle=1.5$. Locations of resonant surfaces, where $q=-m / n$, are indicated.

Figure 2 Positions of the four toroidally separated centrally viewing soft $x$-ray detectors and the 32-chord imaging array.

Figure 3 Time evolution of a central soft $x$-ray signal and reconstructed emission contours for the time period indicated by the dashed lines. Rotation in the $+\theta$ direction is evident. Emission contours are shown in a box of dimensions $-1<x / a<1,-1<y / a<1$.

Figure 4 (a) A phase shift is observed between toroidally separated centrally-viewing SXR signals due to $m=1$ rotation. (b) The 'double frequency' effect observed on chords of small impact parameter is due to the fact that the $m=1$ feature crosses the chord twice per revolution.

Figure $5 \mathrm{~A}$ beating of SXR signals is clearly evident, particularly in the region $r / a=0.3-0.5$. 


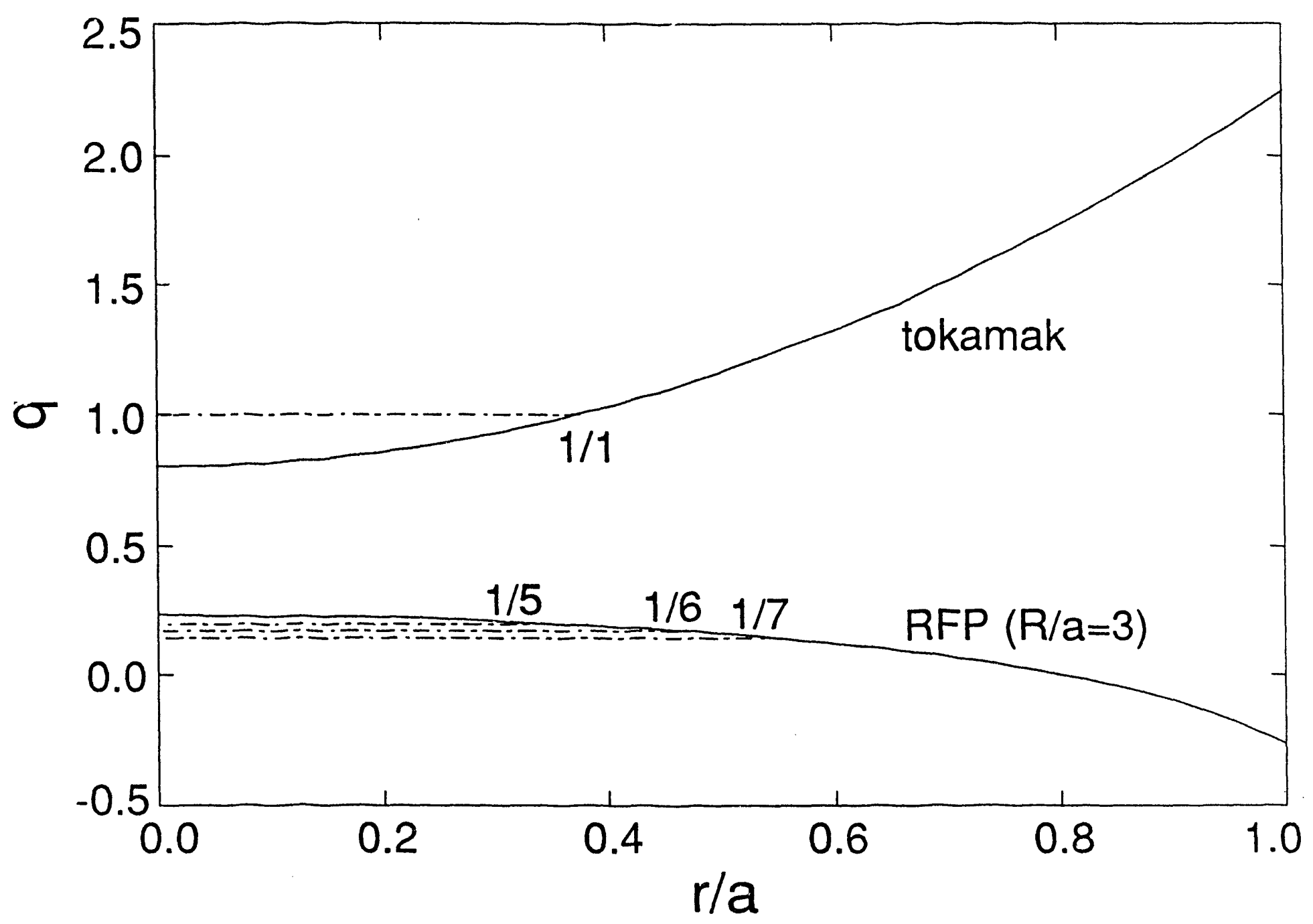

Fig. 1 


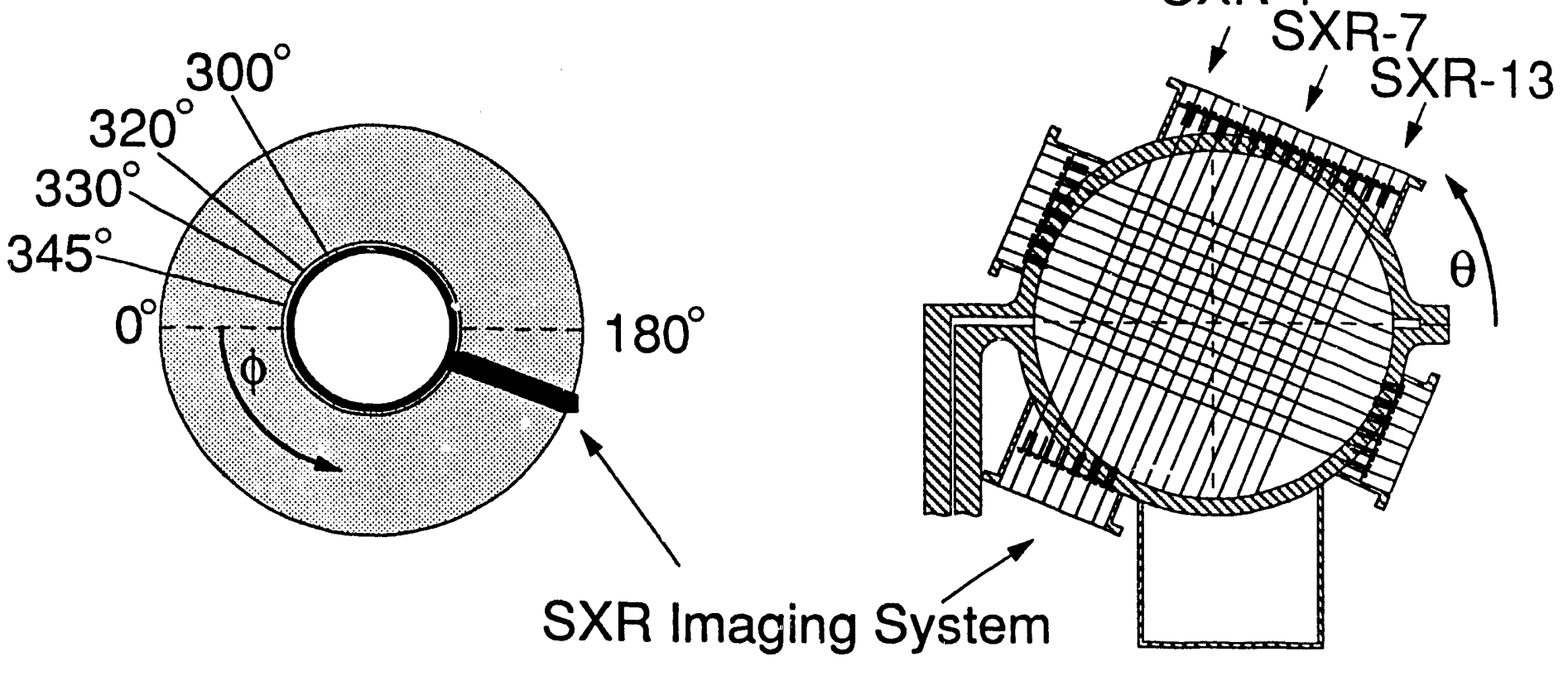

Fig. 2 

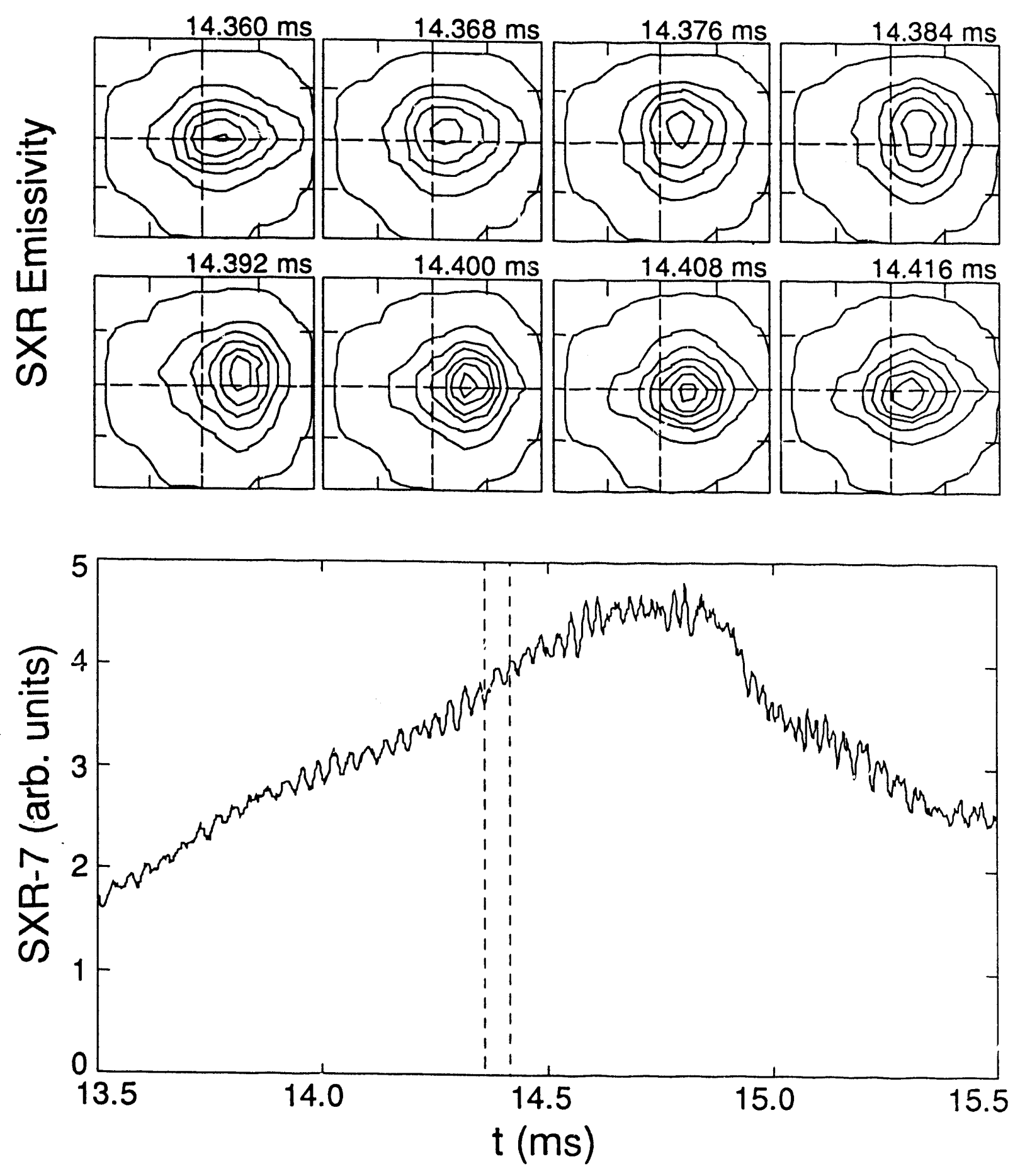

Fig. 3 

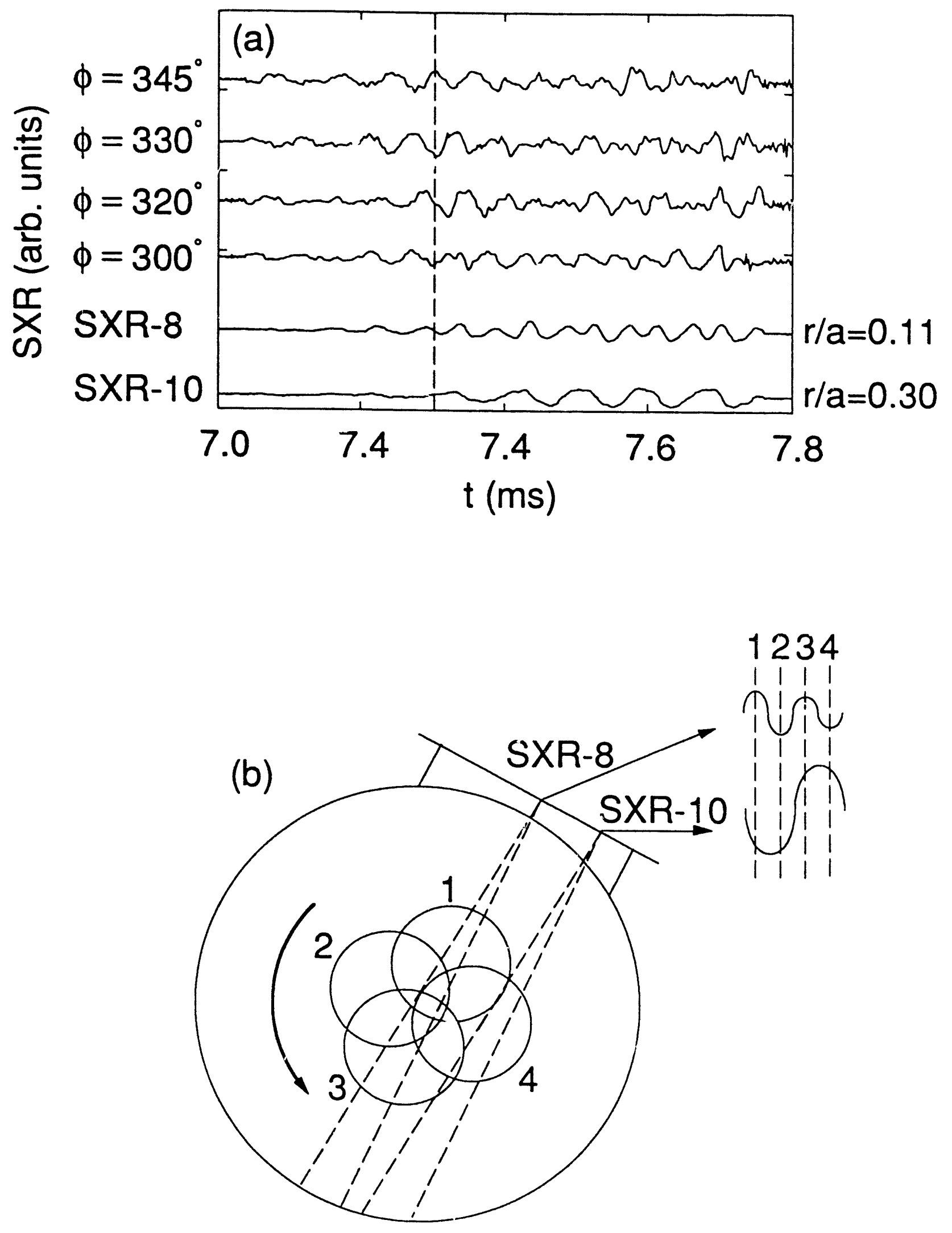

Fig. 4 


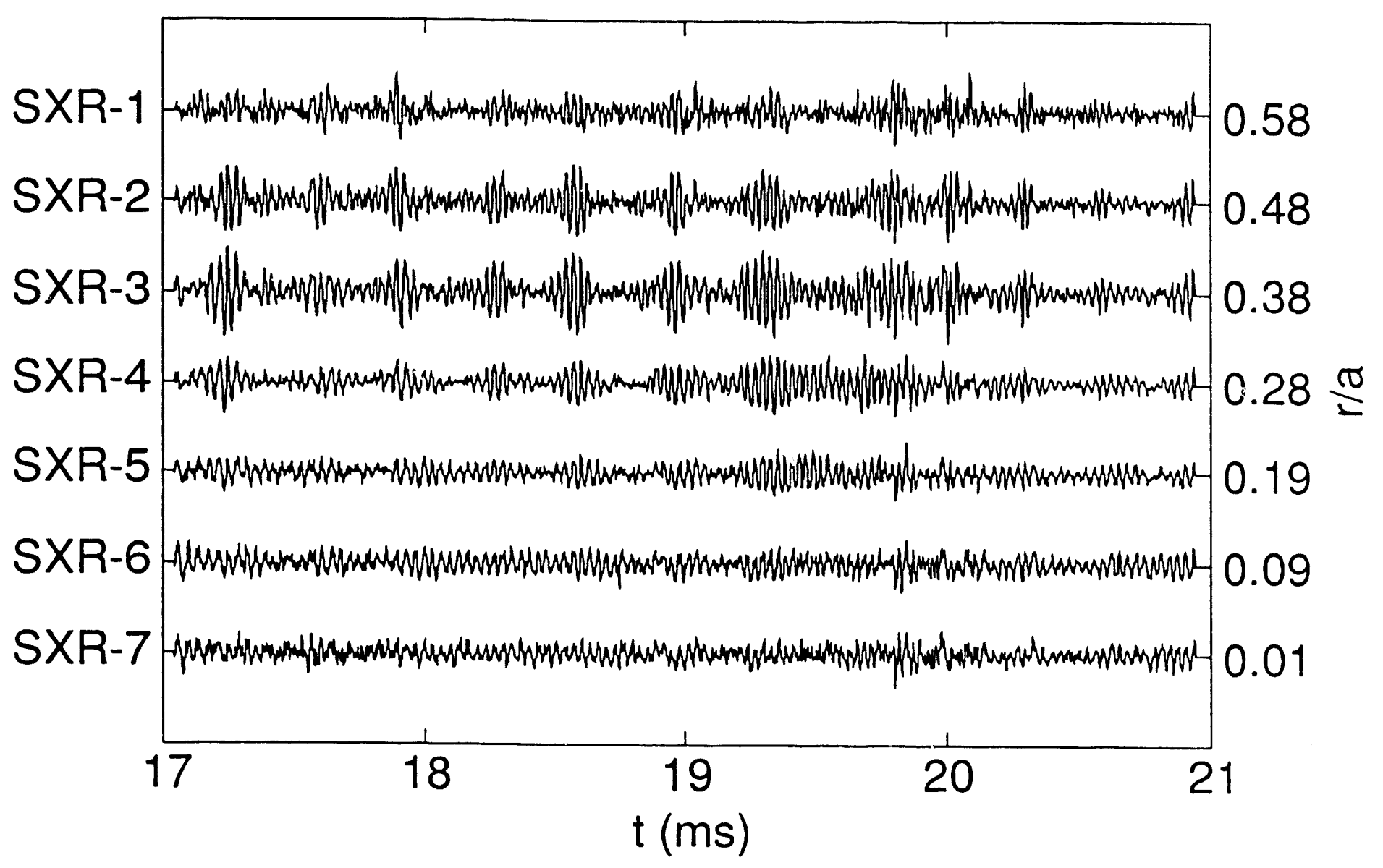




\section{EXTERNAL. DISTRIBUTION IN ADDITION TO UC-20}

S.N. Rasband, Brigham Young University

J.B. Taylor, Institute for Fusion Studies, The University of Texas at Austin

M.A. Abdou, University of California, Los Angeles

R.W. Conn, University of California, Los Angeles

T. Dolan, INEL

R. Smith, University of lowa

F.W. Perkins, PPPL

O. Ishihara, Texas Technical University

P.E. Vandenplas, Association Euratom-Etat Belge, Belgium

Centro Brasileiro de Pesquisas Firicas, Brazil

P. Sakanaka, Institute de Fisica-Unicamp, Brazil

Mme. Monique Bex, GANIL, France

J. Radet, CENCADARACHE, France

University of loannina , Greere

S. Ortolani, Istituto Gas Ionizzati, EURATON-ENEA-CNR Association, Italy

R. Andreani, Associazione EURATOM-ENEA sulla Fusione, Italy

Y. Kondoh, Gunma University, Kiryu, Gunma, Japan

FOM-Instituut voor Plasmafysica "Rijnhuizen", The Netherlands

Z. Ning, Academia Sinica, People's Republic of China

P. Yang, Shandong University, People's Republic of China

S. Zhu, University of Science \& Technology of China, People's Republic of China

I.N. Bogatli, Institute of Atomic Physics, Romania

M.J. Alport, University of Natal, Durban, South Africa

R. Storer, The Flinders University of South Australia, South Australia

B. Lehnert, Royal Institute of Technology, Sweden

Librarian, CRPP, Ecole Polytechnique Federale de Lausanne, Switzerland

B. Alper, Culham Laboratory, UK

A. Newton, Culham Laboratory, UK

6 for Chicago Operations Office

4 for individuals in Washington Offices

INTERNAL DISTRIBUTION IN ADDITION TO UC-20

80 for local group and file 

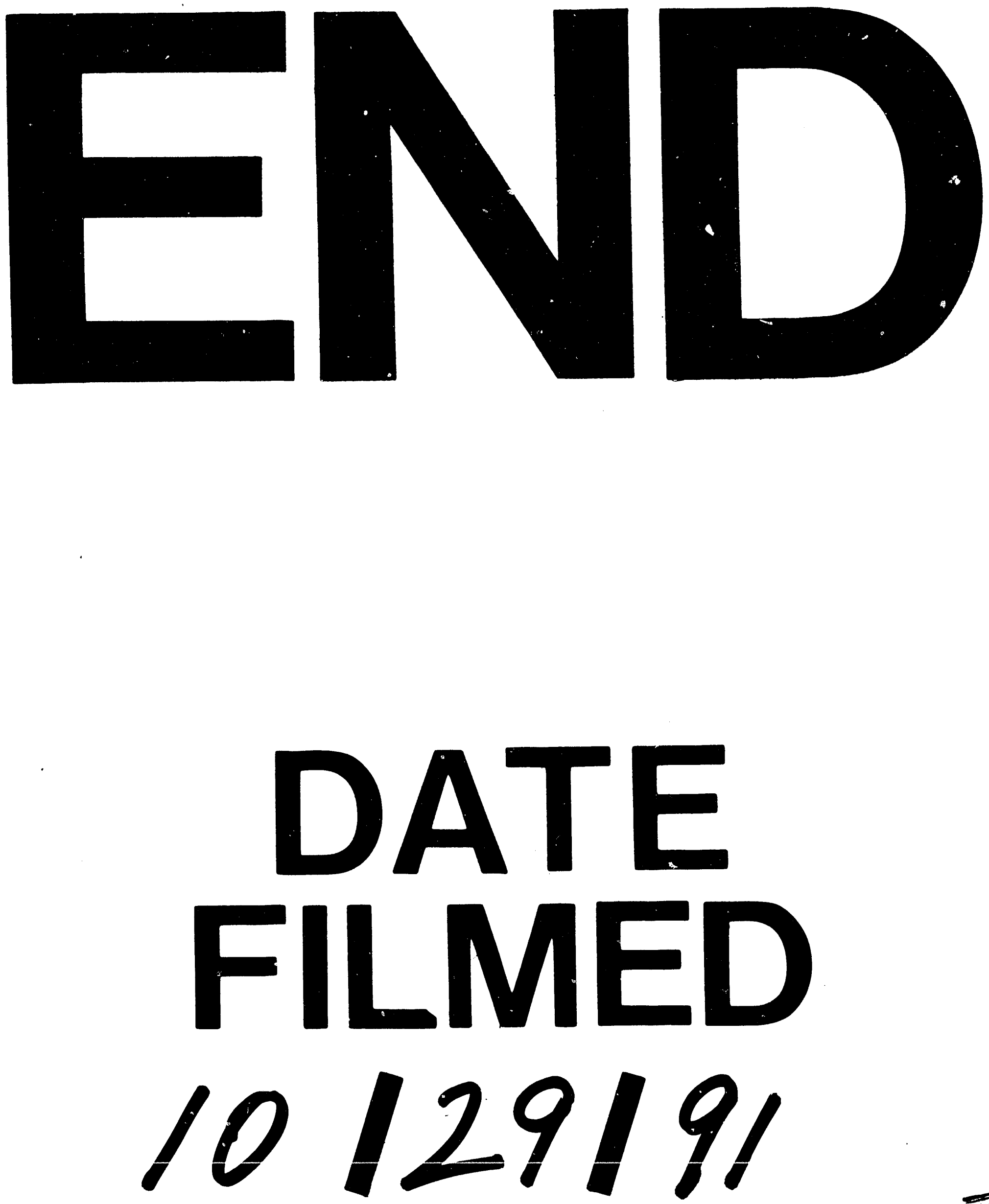

II 


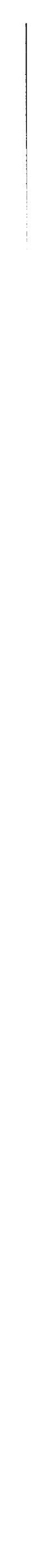

